\title{
Whole-brain CT perfusion imaging using increased sampling intervals: A pilot study
}

\author{
GUOQUAN CAO, WEIJIAN CHEN, HOUZHANG SUN, XIANZHONG GUO, \\ YUNJUN YANG, KUN TANG and JINJIN LIU
}

Department of Radiology, The First Affiliated Hospital of Wenzhou Medical University, Wenzhou, Zhejiang 325000, P.R. China

Received April 13, 2016; Accepted March 17, 2017

DOI: $10.3892 /$ etm.2017.4816

\begin{abstract}
The aim of the present study was to investigate the feasibility of whole-brain perfusion imaging using the increased sampling interval protocol for 320-detector row dynamic-volume computed tomography (CT). A total of 12 volunteers were recruited. The novel protocols with 11 volumes (defined as protocol P11) and 15 volumes (defined as protocol P15) were performed on the volunteers to evaluate whether P11 and P15 are able to acquire comparable results to the standard protocol with 19 volumes (defined as protocol P19) according to the as-low-as-reasonably-achievable principle. All data were acquired using a dynamic-volume CT scanner with a $16 \mathrm{~cm}$-wide detector with 320 rows. The scanned transverse images from volunteers were analyzed using the Volume-Engineered System workstation. The MedCalc software package was used for Bland-Altman analysis of all variables. The data inconsistency of mean transit time (MTT), cerebral blood volume (CBV), cerebral blood flow (CBF), and time to peak (TTP) between $\mathrm{P} 11 / \mathrm{P} 15$ and P19 were all $<5 \%$, and the data were trustworthy. The mean differences of MTT, CBV, CBF and TTP between P15 and P19 were less than those between P11 and P19. The consistencies of perfusion parameters acquired with protocols P15 and P19 were higher compared with those acquired with P11. In whole-brain perfusion, the new protocol P15 has higher consistency with P19 than P11, and the radiation dose may be reduced by $\sim 16 \%$ without degradation of perfusion parameters.
\end{abstract}

Correspondence to: Dr Kun Tang, Department of Radiology, The First Affiliated Hospital of Wenzhou Medical University, 2 Fuxue Lane, Wenzhou, Zhejiang 325000, P.R. China

E-mail: kuntang886@sina.com

Abbreviations: CT, computed tomography; MTT, mean transit time; $\mathrm{CBV}$, cerebral blood volume; $\mathrm{CBF}$, cerebral blood flow; TTP, time to peak; CTP, CT perfusion; ALARA, as-low-as-reasonably-achievable principle; CTA, CT angiography; VES, volume-engineered system; ROI, region of interest; TDC, time-density curve; AE-TDC, arterial enhancement TDC; VE-TDC, venous enhancement TDC; TE-TDC, tissue enhancement TDC

Key words: 320-detector row CT, computed tomography perfusion, radiation dose, sampling interval
Therefore, P15 should be recommended as a routine procedure in whole-brain perfusion imaging.

\section{Introduction}

Dynamic computed tomography (CT) was first used to measure tissue perfusion only 8 years after Sir Geoffrey Hounsfield invented the first head CT (1). However, the perfusion result from the dynamic helical scan is affected by the time difference at different scanning planes. Aquilion ONE of Toshiba Medical Systems is the world's first dynamic-volume CT system. Volumetric scans, with a detector width of $16 \mathrm{~cm}$, enable the entire brain to be scanned in a single gantry rotation. Sequentially acquired volumetric scans provide dynamic volume imaging and may be used in time-based functional studies.

Dynamic-volume imaging of the brain during contrast medium infusion provides true 4D CT digital subtraction angiography images of the intracranial circulation. Using the dynamic-volume imaging method, a number of distinct clinical applications have been generated for intracranial ischemic diseases (2), intracranial aneurysms (3-5), venous anomalies (6), arteriovenous malformations (7-10) and large-vessel vasculitis (11).

However, the radiation dose resulting from dynamic-volume imaging is larger than that of a conventional CT enhancement scan. The Neuro ONE protocol of the Aquilion ONE system is specifically designed for comprehensive stroke assessment of the entire brain in a 60-second scan sequence. Generally, the standard protocol with 19 volumes (defined as protocol P19), which is provided by the vendor, is used for performing brain CT perfusion (CTP), and the corresponding effective dose is $\sim 6 \mathrm{mSv}$. In order to further reduce the radiation dose for CTP according to the as-low-as-reasonably-achievable (ALARA) principle, the purpose of the present study was to investigate the feasibility of new protocols of whole-brain perfusion imaging by using the increased sampling interval protocol, such as the protocol with 15 volumes (defined as protocol P15) and the protocol with 11 volumes (defined as protocol P11).

\section{Materials and methods}

Subjects. The study was approved by the Institutional Research Ethics Committee of the First Affiliated Hospital of Wenzhou 
Medical University (Wenzhou, China), and all participants provided written informed consent prior to the study. From April to December 2015, a total of 12 volunteers participated in the current study (Table I). The volunteers had an age range of 46-73 years, and comprised 6 males and 6 females. All volunteers were randomly assigned to two groups. P11 and P19 protocols were performed in group $1(\mathrm{n}=6)$, and P15 and P19 protocols were performed in group $2(\mathrm{n}=6)$. The inclusion criteria were: i) Age $>45$ years; ii) no symptoms of hemorrhage or ischemia present in the P19 scanning; and iii) a scanning interval of $6 \mathrm{~h}$ between the two protocols, with P19 protocol performed first. The exclusion criteria were: i) History of allergy to iodine-containing contrast medium; ii) unconsciousness; iii) history of stroke; and iv) tumors and other pathologies.

Scanning parameters. All data were acquired using a dynamic-volume $\mathrm{CT}$ scanner with a 16-cm wide detector of 320 rows (Aquilion ONE; Toshiba Medical Systems Corporation, Nasu, Japan) with a bolus of nonionic contrast agent (Omnipaque, $350 \mathrm{mg}$ iodine $/ \mathrm{ml}$, 60-70 ml; GE Healthcare Life Sciences, Chalfont, UK). The contrast agent was injected into the right antecubital vein at a rate of $4.0 \mathrm{ml} / \mathrm{sec}$. The whole brain was scanned for $60 \mathrm{sec}$ using protocols P19, P15 and P11. The reconstruction parameters of these protocols were $0.5 \mathrm{~mm}$ thickness and adaptive iterative dose reduction 3D standard iterative reconstruction. Detailed information of scanning parameters of these protocols is presented in Tables II-IV and Fig. 1.

In the present study, a novel protocol based on the principles of the original P19 protocol was designed (Fig. 1). The mask volume and the volumes for CT angiography (CTA) were scanned using a higher tube current to obtain images with a higher signal-to-noise ratio. The effective radiation dose for brain CT was estimated by multiplying the dose-length product with an established conversion factor of $0.0021 \mathrm{mSv} /(\mathrm{mGy} \cdot \mathrm{cm})$, as has been proposed by the European Working Group for Guidelines on Quality Criteria in CT and endorsed by the American Association of Physicists in Medicine (12).

Imaging analysis. All transverse section data of the volunteers were analyzed using the Volume-Engineered System (VES version 6.4.2) workstation (Vital Solutions; Toshiba Medical Systems Corporation). The deconvolution algorithm was used to generate maps of cerebral blood volume (CBV), cerebral blood flow (CBF), mean transit time (MTT) and time to peak (TTP) and delay time. For each brain, all map data were sampled to 169-188 slices, from the top to the base of the skull, with a sampling interval of $0.5 \mathrm{~mm}$. A total of 18 regions of interest (ROIs) were set for each slice of all of the map images (Fig. 2). The average value and standard deviation were shown adjacent to the corresponding ROI. The data demonstrated in the maps were automatically stored as 'csv' files when the slices changed, which meant that it was not necessary to record data one by one.

The perfusion software in the VES workstation requires three input curves: i) The arterial enhancement time-density curve(AE-TDC); ii) the venous enhancement time-density curve (VE-TDC); and iii) the tissue enhancement time-density curve (TE-TDC). These three time-density curves are the basis of different mathematical models to calculate the hemodynamic parameters of brain tissue.
Table I. Basic information for all subjects.

\begin{tabular}{clcc}
\hline Patient no. & Sex & Age, years & Scanning protocols \\
\hline 1 & Female & 48 & P15 and P19 \\
2 & Male & 71 & P11 and P19 \\
3 & Male & 49 & P11 and P19 \\
4 & Female & 72 & P15 and P19 \\
5 & Male & 69 & P15 and P19 \\
6 & Female & 73 & P11 and P19 \\
7 & Female & 52 & P11 and P19 \\
8 & Male & 63 & P11 and P19 \\
9 & Female & 46 & P15 and P19 \\
10 & Male & 54 & P15 and P19 \\
11 & Male & 50 & P11 and P19 \\
12 & Female & 52 & P15 and p19 \\
\hline
\end{tabular}

Table II. P19 scanning protocol.

\begin{tabular}{lcccccc}
\hline & & & $\begin{array}{c}\text { Rotation } \\
\text { time, } \\
\text { Sec }\end{array}$ & $\begin{array}{c}\text { Sampling } \\
\text { time, } \\
\text { sec }\end{array}$ & $\begin{array}{c}\text { Acquisition } \\
\text { interval, } \\
\text { sec }\end{array}$ & $\begin{array}{c}\text { Total } \\
\text { acquisition } \\
\text { time, sec }\end{array}$ \\
\hline 1 & 80 & 310 & 0.75 & 7 & - & 1 \\
2 & 80 & 150 & 0.75 & $11-17$ & 2 & 6 \\
3 & 80 & 300 & 0.75 & $20-28$ & 2 & 8 \\
4 & 80 & 150 & 0.75 & $31-37$ & 2 & 6 \\
5 & 80 & 150 & 0.75 & $40-60$ & 5 & 20 \\
\hline
\end{tabular}

Table III. P15 scanning protocol.

\begin{tabular}{|c|c|c|c|c|c|c|}
\hline Scan & $\mathrm{kV}$ & $\mathrm{mA}$ & $\begin{array}{l}\text { Rotation } \\
\text { time, } \\
\text { sec }\end{array}$ & $\begin{array}{l}\text { Sampling } \\
\text { time, } \\
\text { sec }\end{array}$ & $\begin{array}{l}\text { Acquisition } \\
\text { interval, } \\
\text { sec }\end{array}$ & $\begin{array}{c}\text { Total } \\
\text { acquisition } \\
\text { time, sec }\end{array}$ \\
\hline 1 & 80 & 310 & 0.75 & 7 & - & 1 \\
\hline 2 & 80 & 150 & 0.75 & $11-17$ & 3 & 6 \\
\hline 3 & 80 & 300 & 0.75 & $20-32$ & 3 & 12 \\
\hline 4 & 80 & 150 & 0.75 & $37-43$ & 3 & 6 \\
\hline 5 & 80 & 150 & 0.75 & $50-60$ & 5 & 10 \\
\hline
\end{tabular}

The perfusion software provides automatic and manual selections of ROI for AE-TDC and VE-TDC. The automatic method was used for all ROIs of AE-TDC and VE-TDC for all data. During the analysis, it was identified that the location of the ROI for AE-TDC or VE-TDC may be different in P11/P15 and P19. This difference may result in a bias for comparison, particularly when using the automatic method for ROI selection. Therefore, the ROIs for AE-TDC and VE-TDC were manually selected at central locations of arterial and venous vessels to keep the locations exactly the same for P19 and P11/P15 when the automatic locations of ROIs were mismatched. 
Table IV. P11 scanning protocol.

\begin{tabular}{|c|c|c|c|c|c|c|}
\hline Scan & $\mathrm{kV}$ & $\mathrm{mA}$ & $\begin{array}{l}\text { Rotation } \\
\text { time, } \\
\text { sec }\end{array}$ & $\begin{array}{l}\text { Sampling } \\
\text { time, } \\
\text { sec }\end{array}$ & $\begin{array}{c}\text { Acquisition } \\
\text { interval, } \\
\text { sec }\end{array}$ & $\begin{array}{c}\text { Total } \\
\text { acquisition } \\
\text { time, sec }\end{array}$ \\
\hline 1 & 80 & 310 & 0.75 & 7 & - & 1 \\
\hline 2 & 80 & 150 & 0.75 & $15-19$ & 4 & 4 \\
\hline 3 & 80 & 300 & 0.75 & $25-37$ & 4 & 12 \\
\hline 4 & 80 & 150 & 0.75 & $43-47$ & 4 & 4 \\
\hline 5 & 80 & 150 & 0.75 & $54-60$ & 6 & 6 \\
\hline
\end{tabular}
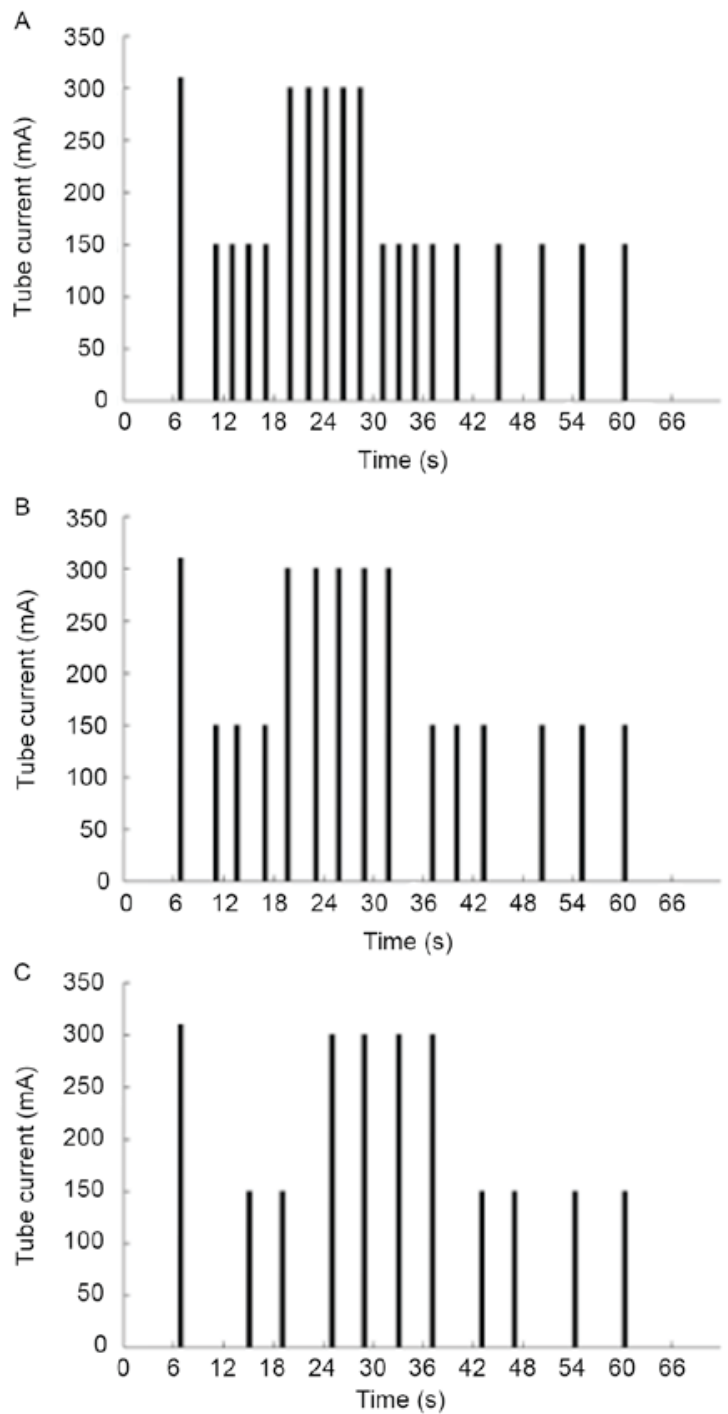

Figure 1. Protocols (A) P19, (B) P15 and (C) P11 are presented. Each black bar represents 1 volume (one phase). All of the dynamic volumes were subdivided into five groups. The first group was used as a mask for the perfusion analysis and the plain scan. The corresponding tube current was $310 \mathrm{~mA}$. The tube current of the third group of dynamic volumes was $300 \mathrm{~mA}$, because images in this group would be used for generating the computed tomographic angiography.

Statistical analysis. The MedCalc version 11.4.2 (MedCalc Software, Ostend, Belgium) statistical software package was used for statistical analysis of all variables. Perfusion parameters were described as the mean and standard deviation. Agreements between the investigation and reference method were evaluated using Bland-Altman analysis (13).

\section{Results}

Effective doses. The effective doses used for all patients in groups 1 and 2 are presented in Table V. The radiation doses in the P11 and P15 protocols were reduced by 36 and 16\%, respectively, compared with those in P19.

Bland-Altman consistency analysis of perfusion parameters, including MTT, $C B V, C B F$ and TTP in groups 1 and 2

Comparison of MTT between P11 and P19. Results indicated that the mean difference in MTT between P11 and P19 was 0.1 , and the majority of the data were distributed in the $95 \%$ confidence interval of -2.7 to 2.8 . The number of data outside the $95 \%$ confidence interval was $895 / 19,116$ (4.68\%). The maximum absolute difference between these two protocols was 9.32 (Table VI; Fig. 3A).

Comparison of $C B V$ between P11 and P19. Results demonstrated that the mean difference in CBV between P11 and P19 was 0.4 , and the majority of the data were distributed in the $95 \%$ confidence interval of -1.6 to 0.9 . The number of data outside the $95 \%$ confidence interval was $635 / 19,116$ (3.32\%). The maximum absolute difference between these two protocols was 8.48 (Table VI; Fig. 3B).

Comparison of CBF between P11 and P19. Results revealed that the mean difference in CBF between P11 and P19 was 5.2, and the majority of the data were distributed in the $95 \%$ confidence interval of -30.8 to 20.4 . The number of data outside the $95 \%$ confidence interval was $331 / 19,116(1.73 \%)$. The maximum absolute difference between these two protocols was 107.4 (Table VI; Fig. 3C).

Comparison of TTP between P11 and P19. Results demonstrated that the mean difference in TTP between these two methods was 1.8, and the majority of the data were distributed in the $95 \%$ confidence interval of -7.6 to 3.9 . The number of data outside the $95 \%$ confidence interval was $766 / 19,116$ (4.01\%). The maximum absolute difference between these two protocols was 48.7 (Table VI; Fig. 3D).

Comparison of MTT between P15 and P19. Results indicated that the mean difference in MTT between P15 and P19 was 0 , and the majority of the data were distributed in the $95 \%$ confidence interval of -1.4 to 1.3 . The number of data outside the $95 \%$ confidence interval was $812 / 19,332$ (4.20\%). The maximum absolute difference between these two protocols was 6.95 (Table VI; Fig. 3E).

Comparison of $C B V$ between P15 and P19. Results revealed that the mean difference in CBV between these two methods was 0 , and the majority of the data were distributed in the $95 \%$ confidence interval of -1.2 to 1.2 . The number of data outside the $95 \%$ confidence interval was $344 / 19,332$ (1.78\%). The maximum absolute difference between these two protocols was 10.91 (Table VI; Fig. 3F). 


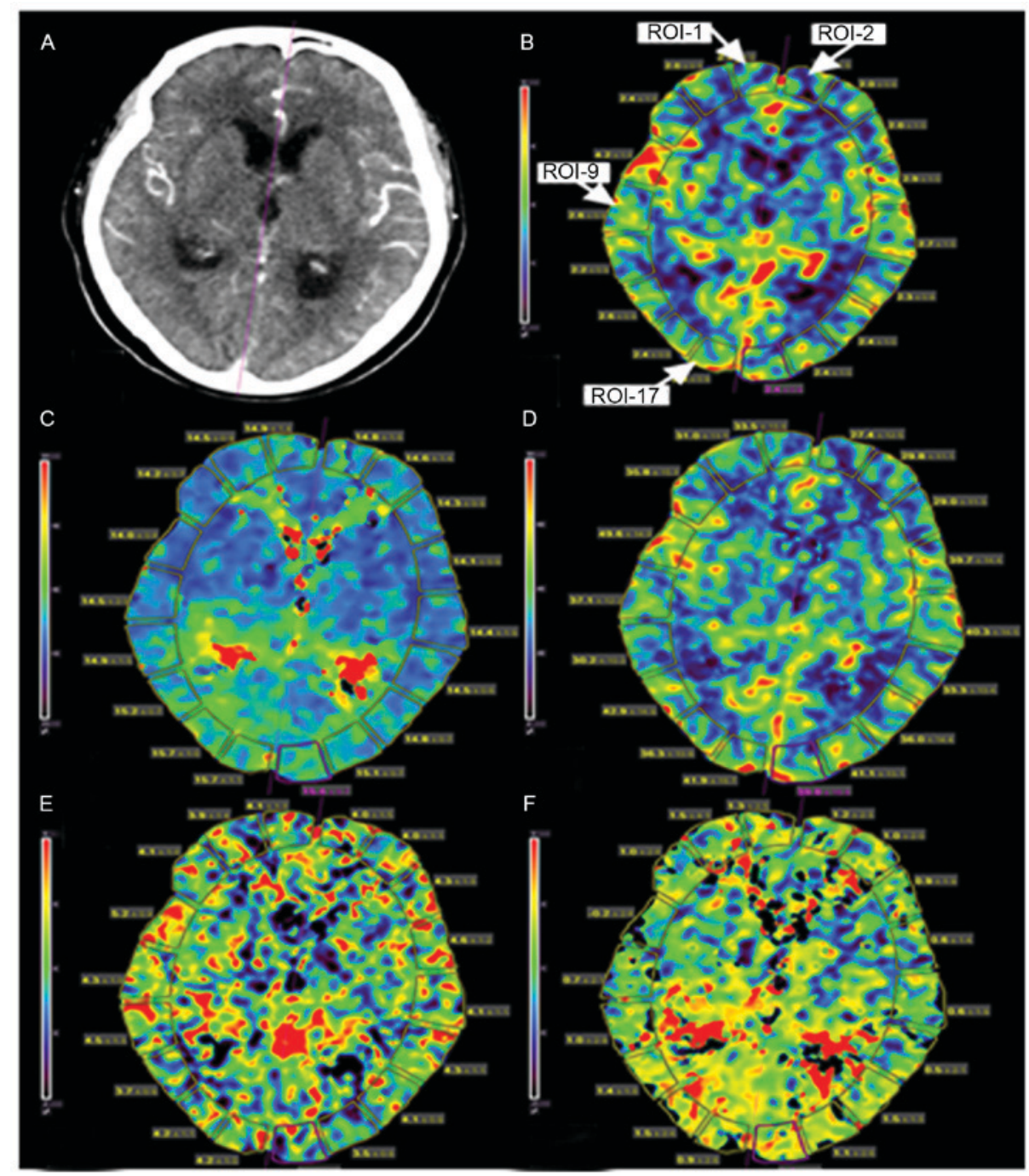

Figure 2. Sampling slice and maps of the assessed parameter for each slice. (A) Sampling slice at the level of basal ganglia. Maps of (B) cerebral blood volume, (C) time to peak, (D) cerebral blood flow, (E) mean transit time and (F) delay time. The eighteen small dot-containing labels around the edge of each brain slice indicated the ROIs. The distribution order of 18 ROIs in each brain slice was as follow: ROI-1 and ROI-2 were respectively distributed at the front left and front right of each slice, ROI-3 and ROI-4 were respectively distributed at the behind of ROI- 1 and ROI-2, and so on. Four of the ROIs are indicated by white arrows in (B) ROI, region of interest.

Comparison of CBF between P15 and P19. Results demonstrated that the mean difference in CBV between P15 and P19 was 0.1 , and the majority of the data were distributed in the $95 \%$ confidence interval of -19.3 to 19.6 . The number of data outside the $95 \%$ confidence interval was 568/19,332 (2.94\%). The maximum absolute difference between these two protocols was 147.66 (Table VI; Fig. 3G).

Comparison of TTP between P15 and P19. Results indicated that the mean difference in TTP between P15 and P19 was 0.5 , and the majority of the data were distributed in the $95 \%$ confidence interval of -3.6 to 4.7 . The number of data outside the $95 \%$ confidence interval was $738 / 19,332$ (3.82\%). The maximum absolute difference between these two protocols was 38.7 (Table VI; Fig. 3H).

\section{Discussion}

A protocol using 22 volumes (defined as protocol P22) has been used for brain perfusion scanning in a number of previous studies $(14,15)$. Previous studies using the Neuro ONE protocols for various types of subjects using dynamic-volume CT are summarized in Table VII. The majority of the studies performed CTA using the P22 brain perfusion protocol, with the same tube current during the dynamic procedure. The novel Neuro ONE protocol recommended by the vendor was optimized in the CTA phases (volumes) of the present study by increasing the tube current during the arterial phases (Fig. 1). Furthermore, the number of volumes required for the calculation for CT perfusion was reduced from 22 to 19 .

A previous study indicated that the total effective dose was decreased by the reduction of volumes (16). The present study investigated the hypothesis that the dose may be decreased by further reducing the number of volumes. P15 and P11, two novel protocols, were developed to reduce the effective dose for patients. Compared with the standard P19 protocol, the radiation doses were reduced by $\sim 36$ and $16 \%$ in $\mathrm{P} 11$ and $\mathrm{P} 15$, respectively.

In the present study, the consistency of perfusion parameters acquired using different scanning protocols in groups 1 and 2, 
Table V. Effective dose information for all 12 patients.

\begin{tabular}{rccccc}
\hline $\begin{array}{l}\text { Patient } \\
\text { no. }\end{array}$ & $\begin{array}{c}\text { Scanning } \\
\text { protocols }\end{array}$ & $\begin{array}{c}\text { P11 effective } \\
\text { dose, } \mathrm{mSv}\end{array}$ & $\begin{array}{c}\text { P15 effective } \\
\text { dose, } \mathrm{mSv}\end{array}$ & $\begin{array}{c}\text { P19 effective } \\
\text { dose, mSv }\end{array}$ & $\begin{array}{c}\text { Dose reduction } \\
\text { rate, } \%\end{array}$ \\
\hline 1 & P15 and P19 & - & 5.73 & 6.82 & 16.0 \\
2 & P11 and P19 & 4.37 & - & 6.83 & 36.0 \\
3 & P11 and P19 & 4.37 & - & 6.83 & 36.0 \\
4 & P15 and P19 & - & 5.73 & 6.83 & 16.1 \\
5 & P15 and P19 & - & 5.74 & 6.84 & 3.83 \\
6 & P11 and P19 & 4.37 & - & 6.83 & 36.0 \\
7 & P11 and P19 & 4.37 & - & 6.83 & 36.0 \\
8 & P11 and P19 & 4.37 & - & 5.74 & 6.83 \\
9 & P15 and P19 & - & 5.73 & 6.83 & 16.1 \\
10 & P15 and P19 & - & - & 6.84 & 36.0 \\
12 & P11 and P19 & 4.37 & 5.74 & 16.1 \\
\hline
\end{tabular}

Table VI. Comparison of the results between the P11/P15 and P19 protocols.

\begin{tabular}{|c|c|c|}
\hline Perfusion parameters & Group 1 (P11 vs. P19) & Group 2 (P15 vs. P19) \\
\hline \multicolumn{3}{|l|}{ Mean transit time } \\
\hline Confidence interval/mean difference & -2.7 to $2.8 / 0.1$ & -1.4 to $1.3 / 0$ \\
\hline Non-consistent data, \% (n/N) & $4.68(895 / 19,116)$ & $4.20(812 / 19,332)$ \\
\hline Absolute value of the maximum difference & 9.32 & 6.95 \\
\hline \multicolumn{3}{|l|}{ Cerebral blood volume } \\
\hline Confidence interval/mean difference & -1.6 to $0.9 / 0.4$ & -1.2 to $1.2 / 0$ \\
\hline Non-consistent data, $\%(\mathrm{n} / \mathrm{N})$ & $3.32(635 / 19,116)$ & $1.78(344 / 19,332)$ \\
\hline Absolute value of the maximum difference & 8.48 & 10.91 \\
\hline \multicolumn{3}{|l|}{ Cerebral blood flow } \\
\hline Confidence interval/mean difference & -30.8 to $20.4 / 5.2$ & -19.3 to $19.6 / 0.1$ \\
\hline Non-consistent data, \% (n/N) & $1.73(331 / 19,116)$ & $2.94(568 / 19,332)$ \\
\hline Absolute value of the maximum difference & 107.4 & 147.66 \\
\hline \multicolumn{3}{|l|}{ Time to peak } \\
\hline Confidence interval/mean difference & -7.6 to $3.9 / 1.8$ & -3.6 to $4.7 / 0.5$ \\
\hline Non-consistent data, $\%(\mathrm{n} / \mathrm{N})$ & $4.01(766 / 19,116)$ & $3.82(738 / 19,332)$ \\
\hline Absolute value of the maximum difference & 48.7 & 38.7 \\
\hline
\end{tabular}

namely MTT, CBV, CBV and TTP, was evaluated using the Bland-Altman statistical method. The delay time was not used for statistical evaluation as it is only a correction parameter. The results of the current study indicated that the inconsistency rates of MTT, CBV, CBF and TTP data in groups 1 and 2 (i.e., data outside of the $95 \%$ confidence interval) were all $<5 \%$. Therefore, the data are trustworthy. All the mean differences of perfusion parameters in groups 1 and 2, with the exception of MTT and $\mathrm{CBV}$ in group 2, were not 0 , which indicates that differences in perfusion parameters were measured using different scanning protocols and the different values reflected the resulting difference measured using the two scanning protocols. For the results, the mean differences in $\mathrm{MTT}, \mathrm{CBV}, \mathrm{CBF}$ and TTP in group 2 were less than those in group 1, which indicated that the results obtained using P15 were most similar to P19. Therefore, the consistency of the perfusion parameters acquired by P15 and P19 was higher than that of P11 and P19. Furthermore, the differences in perfusion parameters in group 2 (absolute value of the maximum difference) were clinically acceptable. There was limited difference between the mean values of CBF and TTP measured using protocols P15 and P19 (the mean differences of CBF and TTP were 0.1 and 0.5 , respectively), and there are several possible reasons for this. Firstly, the actual artery time to peak may be missed in the third sequence acquired at a high current due to the increasing acquisition time interval of the P15 scanning protocol. Secondly, examinations using P15 and P19 were not performed at exactly the same time. Although the examination time interval was only $6 \mathrm{~h}$, the heart rate and contractility during the corresponding examination sequences may have been different, which may have led to 
Table VII. Comparison of the effective dose in previous studies using the Neuro ONE protocol for brain imaging using dynamic-volume computed tomography.

\begin{tabular}{llccccc}
\hline Author, year & \multicolumn{1}{c}{ Subjects } & $\mathrm{kVp}$ & $\mathrm{mAs}$ & Volumes & $\begin{array}{c}\text { Mean effective } \\
\text { dose, } \mathrm{mSV}\end{array}$ & (Refs.) \\
\hline Krings et al, 2009 & Aneurysm & 80 & 120 & 22 & 3.6 & $(5)$ \\
Yahyavi-Firouz-Abadi et al, 2009 & Vasculitis & 80 & 100 & 18 & 4.6 & $(11)$ \\
Shinohara et al, 2010 & Ischemia & 80 & 150 & $\mathrm{NS}$ & 6.8 & $(2)$ \\
Brouwer et al, 2010 & AVM & 80 & 120 & 22 & 4.0 & $(8)$ \\
Hanson et al, 2011 & Venous anomalies & 80 & 100 & 22 & 4.4 & $(6)$ \\
Willems et al, 2011 & AVM & 80 & 120 & 22 & 5.2 & $(7)$ \\
Willems et al, 2012 & AVM & 80 & 120 & 22 & 5.2 & $(9)$ \\
Hayashida et al, 2013 & Aneurysm & 120 & $\mathrm{NS}$ & $\mathrm{NS}$ & 3.1 & $(13)$ \\
Emmer et al, 2014 & Ischemia & 80 & $97.5 / 120 / 225$ & 18 & 7.3 & $(14)$ \\
\hline
\end{tabular}

NS, not stated; AVM, arteriovenous malformations.
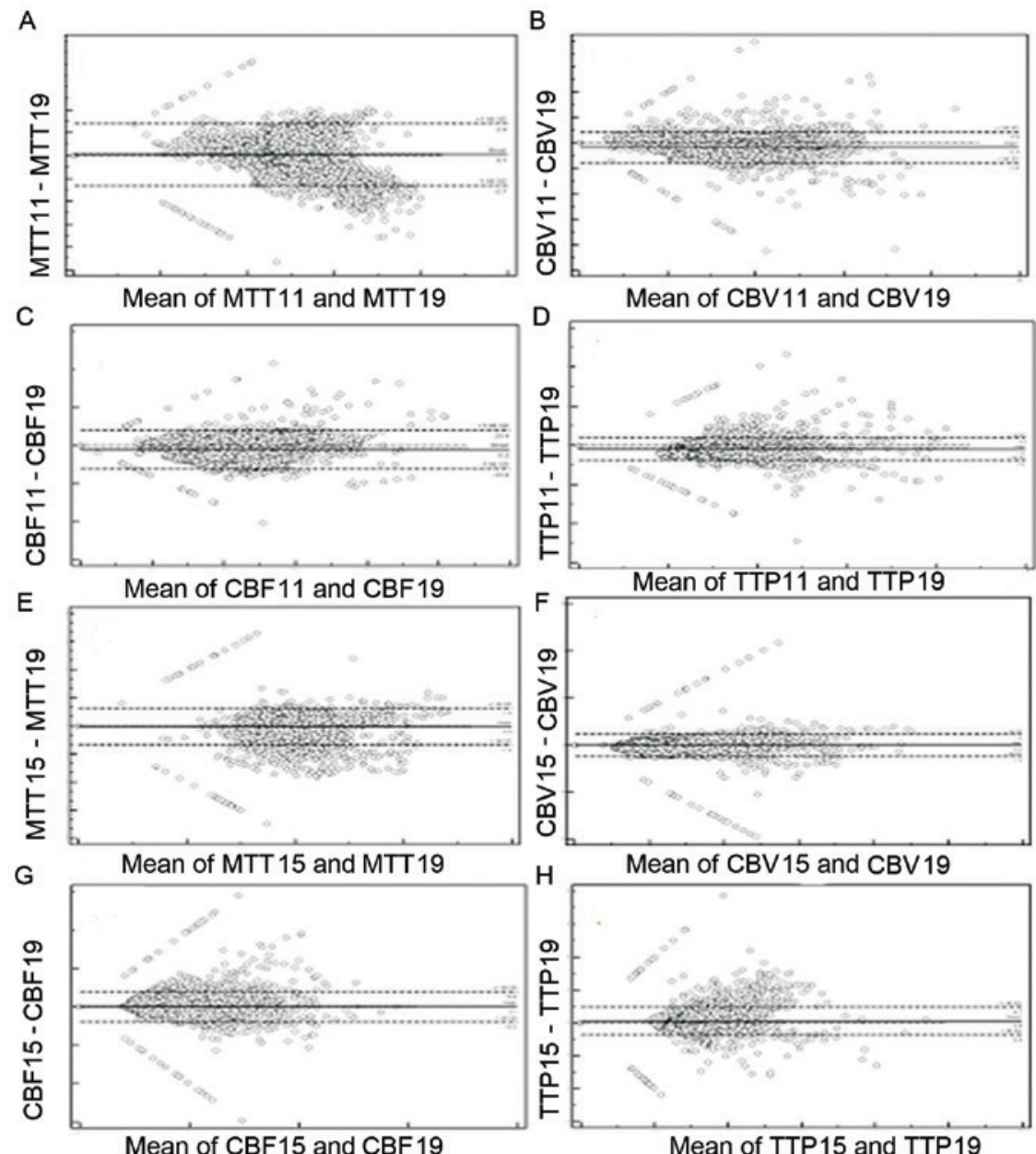

Figure 3. Comparison of Bland-Altman consistency. Results comparing the consistency between P11/P15 and P19 are shown. The consistency between P11 and P19 for the maps of (A) MTT, (B) CBV, (C) CBF and (D) TTP is presented. The consistency between P15 and P19 for the maps of (E) MTT, (F) CBV, (G) CBF and (H) TTP is shown. MTT, mean transit time; CBV, cerebral blood volume; CBF, cerebral blood flow; TTP, time to peak. The numbers 11,15 and 19 following each variable name indicate that the P11, P15 and P19 protocols, respectively, were used.

small differences in brain hemodynamics. Finally, the number of subjects in the current study was limited, which may have increased the measuring errors.
Therefore, it is considered that the results obtained from P15 are not significantly different from that of P19 and the radiation dose would be further reduced by $\sim 16 \%$ with use of 
the P15 protocol. Table VII indicates the effective doses in studies using the old Neuro ONE protocols for various subjects using dynamic-volume CT. The average effective doses were in the range of 3.1 and $7.3 \mathrm{mSv}$. The majority of these studies used a voltage of $80 \mathrm{kVp}$ and a constant tube current in the dynamic volumes. However, the higher tube current was used during the arterial phases in the present study for obtaining an improved signal-to-noise ratio, causing the effective dose to be higher than that in the previous studies.

Shankar et al (17) studied whole-brain perfusion imaging with a 320-detector row dynamic-volume CT scanner. The radiation dose was reduced by increasing the sampling interval in the study. The analysis was simulated using post-processing software, all cases were normal and had no ischemia focus. The old Neuro ONE brain perfusion protocol provided by the vendor was used and the tube current for all the dynamic volumes was the same (17). In the present study, one of the two novel protocols was performed alongside the standard protocol on the same patient. Therefore, the results obtained from the present study are more reliable than those from previous studies. Although two procedures may incur a higher radiation dose for volunteers, all the scanning protocols strictly adhered to the principles of the Neuro ONE scanning protocol and ALARA.

As target arteries in the brain may be affected by the partial-volume effect, the venous time-density curve (TDC) was used to correct the height of the arterial TDC. During the measurement, the ROIs were manually set for P11/P15 and P19 to ensure that the ROIs were selected at the same level and location between P11/P15 and P19. This was a very important step to guarantee comparability. In addition, it was important to provide specific information to the subjects, since they were undergoing perfusion scanning twice; the details, including the radiation damage to the volunteers and the benefit to others were explained.

The limitation of this study was that only 12 patients were studied. However, for each brain, all the map data was sampled to $169-188$ slices, from the top to the base of the skull, and 18 ROIs were set for each slice of all the map images. Therefore, the amount of data used for comparison was very large, which would reduce the data error to a certain extent. A further study using a large number of patients is required to verify the efficacy of this protocol in specific patients with stroke.

In conclusion, for whole-brain perfusion, the novel P15 protocol has a higher consistency with P19 than does P11, and the radiation dose may be reduced by $\sim 16 \%$ without degradation of perfusion parameters. Therefore, $\mathrm{P} 15$ may be recommended as a routine procedure in whole-brain perfusion imaging.

\section{Acknowledgements}

This study was sponsored by a grant from the Zhejiang Provincial Natural Science Foundation of China (LY15H030016).

\section{References}

1. Axel L: Cerebral blood flow determination by rapid-sequence computed tomography: Theoretical analysis. Radiology 137: 679-686, 1980
2. Shinohara Y, Ibaraki M, Ohmura T, Sugawara S, Toyoshima H, Nakamura K, Kinoshita F and Kinoshita T: Whole-brain perfusion measurement using 320-detector row computed tomography in patients with cerebrovascular steno-occlusive disease: Comparison with 15O-positron emission tomography. J Comput Assist Tomogr 34: 830-835, 2010.

3. Luo Z, Wang D, Sun X, Zhang T, Liu F, Dong D, Chan NK and Shen B: Comparison of the accuracy of subtraction CT angiography performed on 320-detector row volume CT with conventional CT angiography for diagnosis of intracranial aneurysms. Eur J Radiol 81: 118-122, 2012.

4. Hayakawa M, Maeda S, Sadato A, Tanaka T, Kaito T, Hattori N, Ganaha T, Moriya S, Katada K, Murayama K, et al: Detection of pulsation in ruptured and unruptured cerebral aneurysms by electrocardiographically gated 3-dimensional computed tomographic angiography with a 320-row area detector computed tomography and evaluation of its clinical usefulness. Neurosurgery 69: 843-851, 2011.

5. Krings T, Willems P, Barfett J, Ellis M, Hinojosa N, Blobel J and Geibprasert S: Pulsatility of an intracavernous aneurysm demonstrated by dynamic 320-detector row CTA at high temporal resolution. Cent Eur Neurosurg 70: 214-218, 2009.

6. Hanson EH, Roach CJ, Ringdahl EN, Wynn BL, DeChancie SM, Mann ND, Diamond AS and Orrison WW Jr: Developmental venous anomalies: Appearance on whole-brain CT digital subtraction angiography and CT perfusion. Neuroradiology 53: 331-341, 2011.

7. Willems PW, Brouwer PA, Barfett JJ, terBrugge KG and Krings T: Detection and classification of cranial dural arteriovenous fistulas using 4D-CT angiography: Initial experience. AJNR Am J Neuroradiol 32: 49-53, 2011.

8. Brouwer PA, Bosman T, Van Walderveen MA, Krings T, Leroux AA and Willems PW: Dynamic 320-section CT angiography in cranial arteriovenous shunting lesions. AJNR Am J Neuroradiol 31: 767-770, 2010.

9. Willems PW, Taeshineetanakul P, Schenk B, Brouwer PA, Terbrugge KG and Krings T: The use of 4D-CTA in the diagnostic work-up of brain arteriovenous malformations. Neuroradiology 54: 123-131, 2012.

10. Kim DJ and Krings T: Whole-brain perfusion CT patterns of brain arteriovenous malformations: A pilot study in 18 patients. AJNR Am J Neuroradiol 32: 2061-2066, 2011.

11. Yahyavi-Firouz-Abadi N, Wynn BL, Rybicki FJ, Steigner ML, Hussain AZ, Mather R, Hanson EH, Ansarinia M and Orrison WW Jr: Steroid-responsive large vessel vasculitis: Application of whole-brain 320-detector row dynamic volume $\mathrm{CT}$ angiography and perfusion. AJNR Am J Neuroradiol 30: 1409-1411, 2009.

12. Bongartz G, Golding SJ, Jurik AG, Leonardi M, van Persijn van Meerten E, Rodríguez R, Schneider K, Calzado A, Geleijns J, Jessen KA, et al: European Guidelines for Multislice Computed Tomography. European Commission, 2004.

13. Bunce C: Correlation, agreement, and Bland-Altman analysis: Statistical analysis of method comparison studies. Am J Ophthalmol 148: 4-6, 2009.

14. Hayashida E, Sasao A, Hirai T, Hamasaki K, Nishi T, Utsunomiya D, Oda S, Iryo Y, Urata J and Yamashita Y: Can sufficient preoperative information of intracranial aneurysms be obtained by using 320-row detector CT angiography alone? Jpn J Radiol 31: 600-607, 2013.

15. Emmer BJ, Rijkee M, Niesten JM, Wermer MJ, Velthuis BK and van Walderveen MA: Whole brain CT perfusion in acute anterior circulation ischemia: Coverage size matters. Neuroradiology 56: 1121-1126, 2014

16. Fu J, Chen WJ, Wu GY, Cheng JL, Wang MH, Zhuge Q, Li JC, Zhang Q, Zhang Y, Xia NZ and Yang YJ: Whole-brain 320-detector row dynamic volume CT perfusion detected crossed cerebellar diaschisis after spontaneous intracerebral hemorrhage. Neuroradiology 57: 179-187, 2015.

17. Shankar JJ, Lum C and Sharma M: Whole-brain perfusion imaging with 320-MDCT scanner: Reducing radiation dose by increasing sampling interval. AJR Am J Roentgenol 195: 1183-1186, 2010. 